\title{
Corrigendum: Mutational signatures in esophageal adenocarcinoma define etiologically distinct subgroups with therapeutic relevance
}

Maria Secrier, Xiaodun Li, Nadeera de Silva, Matthew D Eldridge, Gianmarco Contino, Jan Bornschein, Shona MacRae, Nicola Grehan, Maria O’Donovan, Ahmad Miremadi, Tsun-Po Yang, Lawrence Bower, Hamza Chettouh, Jason Crawte, Núria Galeano-Dalmau, Anna Grabowska, John Saunders, Tim Underwood, Nicola Waddell, Andrew P Barbour, Barbara Nutzinger, Achilleas Achilleos, Paul A W Edwards, Andy G Lynch, Simon Tavaré \& Rebecca C Fitzgerald on behalf of the Oesophageal Cancer Clinical and Molecular Stratification (OCCAMS) Consortium

Nat. Genet.; doi:10.1038/3659; corrected online 19 September 2016

In the version of this article initially published online, the mutation signature illustrations for S1 and S2 in Figure 3a were switched. Additionally, in the Online Methods, the text originally stated that structural variants were called using BWA-MEM, when it should have stated that these were called using BWA. These errors have been corrected for the print, PDF and HTML versions of this article.

\section{Corrigendum: Mutations in SNORD118 cause the cerebral microangiopathy leukoencephalopathy with calcifications and cysts}

Emma M Jenkinson, Mathieu P Rodero, Paul R Kasher, Carolina Uggenti, Anthony Oojageer, Laurence C Goosey, Yoann Rose, Christopher J Kershaw, Jill E Urquhart, Simon G Williams, Sanjeev S Bhaskar, James O'Sullivan, Gabriela M Baerlocher, Monika Haubitz, Geraldine Aubert, Kristin W Barañano, Angela J Barnicoat, Roberta Battini, Andrea Berger, Edward M Blair, Janice E Brunstrom-Hernandez, Johannes A Buckard, David M Cassiman, Rosaline Caumes, Duccio M Cordelli, Liesbeth M De Waele, Alexander J Fay, Patrick Ferreira, Nicholas A Fletcher, Alan E Fryer, Himanshu Goel, Cheryl A Hemingway, Marco Henneke, Imelda Hughes, Rosalind J Jefferson, Ram Kumar, Lieven Lagae, Pierre G Landrieu, Charles M Lourenço, Timothy J Malpas, Sarju G Mehta, Imke Metz, Sakkubai Naidu, Katrin Õunap, Axel Panzer, Prab Prabhakar, Gerardine Quaghebeur, Raphael Schiffmann, Elliott H Sherr, Kanaga R Sinnathuray, Calvin Soh, Helen S Stewart, John Stone, Hilde Van Esch, Christine E G Van Mol, Adeline Vanderver, Emma L Wakeling, Andrea Whitney, Graham D Pavitt, Sam Griffiths-Jones, Gillian I Rice, Patrick Revy, Marjo S van der Knaap, John H Livingston, Raymond T O’Keefe \& Yanick J Crow

Nat. Genet.; doi:10.1038/ng.3661; corrected online 19 September 2016

In the version of this article initially published, the names of authors Geraldine Aubert, Gerardine Quaghebeur and Yoann Rose were misspelled. The error has been corrected in the print, HTML and PDF versions of the article.

Corrigendum: Integrative genomic analysis implicates limited peripheral adipose storage capacity in the pathogenesis of human insulin resistance

Luca A Lotta, Pawan Gulati, Felix R Day, Felicity Payne, Halit Ongen, Martijn van de Bunt, Kyle J Gaulton, John D Eicher, Stephen J Sharp, Jian'an Luan, Emanuella De Lucia Rolfe, Isobel D Stewart, Eleanor Wheeler, Sara M Willems, Claire Adams, Hanieh Yaghootkar, EPIC-InterAct Consortium, Cambridge FPLD1 Consortium, Nita G Forouhi, Kay-Tee Khaw, Andrew D Johnson, Robert K Semple, Timothy Frayling, John R B Perry, Emmanouil Dermitzakis, Mark I McCarthy, Inês Barroso, Nicholas J Wareham, David B Savage, Claudia Langenberg, Stephen O'Rahilly \& Robert A Scott

Nat. Genet.; doi:10.1038/ng.3714; corrected online 5 December 2016

In the version of this article initially published online, the middle initial of collaborator Maarten R. Soeters was inadvertently omitted. The error has been corrected for the print, PDF and HTML versions of this article. 